\title{
Aménagement du Rhin de Bâle à Strasbourg et à Lauterbourg au moyen d'une Méthode nouvelle de canalisation des Fleuves

\author{
Pour la production de la force motrice, la navigation internationale, l'irrigation des deux rives \\ et la protection contre les inondations
}

par I. MAHI, Ingénieur

\section{DISPOSITIONS TECHNIQUES RELATIVES A L'ÉTABLISSEMENT DES BARRAGES-USINES}

Les emplacements les plus propices sont choisis a l'amont des confluents et autant que possible sur le prolongement de grandes voies transversales auxquelles on offrira des ponts confortables remplaçant les ponts de bateaux actuels.

On aura intérêt à espacer les digues le plus possible, quand on n'aura pas de sacrifice de surfaces coiteuses à faire, en vue de réaliser des biefs de très grandes capacités : en temps de crues, la vitesse de l'eau s'accusera davantage dans leur partie centrale approfondie gràce à la majoration, sur ce point, du rayon moyen : d'où circulation normale des alluvions et empèchement des atterrissements, qu'on combattra comme on l'a fait avec succès sur le Danube à l'aide des hersages si économiques, alors surtout qu'on disposera du halage électrique assurant l'action énergique d'un bateau herseur.

Pendant les crues, les bateaux trouveront une moindre vitesse en se tenant proche des rives. En temps d'eaux moyennes et d'étiage, le débit, à l'heure de la pointe, par exemple, devant ètre majoré sur toutes les usines, on aura une circulation générale d'autant moins accélérée et une perte de charge d'autant plus réduite que la section des biefs sera plus grande.

Lorsque la largeur le permettra, on mettra l'usine, par économie de longueur des barrages, dans le prolongement de l'ouvrage de décharge. Le canal à ménager entre la rive et un ilot ou une estacade assurant une eau calme à l'accès des bateaux aux écluses, sera sur la rive droite, alors que l'usine sera sur la rive gauche avec un canal de fuite le plus court possible. Les écluses établies à l'amont, les ponts n'auront pas à être relevés pour favoriser le tirant d'air aux bateaux, puisque ces derniers seront au niveau du bief intérieur en entrant ou en sorțant des écluses vers l'aval. Les portes de ces écluses seront du type Stonay : pour mieux assurer le retour d'eau sous les bateaux pendant les mouvements de translation dans les écluses, on assurera à ces dernières une surprofondeur de mouillage.

L'intérêt à accorder aux moyens de protection contre les infiltrations ne se justifiera en dépense qu'à partir de $5 \%$ environ d'économie d'eau. Envisageons en conséquence la solution de l'enfoncement longitudinal sous la digue de palplanches métalliques à coulisses. On les réalisera avec noyau creux pour assurer ensuite, par une injection en place, une étanchéité complémentaire, une fois le voile réalisé (fig. 14 et 15 ).

Si ces palplanches représentent un poids de 75 kilos par mètre superficiel, et que leur enfoncement au mouton, extrêmement rapide avec l'outillage voulu, en porte le prix à 20 franes or ; alors même qu'on reconnaîtrait la nécessité d'effectuer ce voile sur les quatre cinquièmes des deux rives, soit sur 200 kilomètres, la dépense serait de l'ordre de 20 millions, représentant au plus 1.400 .000 francs par an de dépense, capital.

En épargnant de l'eau, pouvant produire des kilowatts-heure d'une valeur brute de 0 fr. 05 or, il suffira de récupérer 28 millions de kilowatts-heure pour couvrir les frais de protection contre les infiltrations. Mais ce chiffre, sur plus de 8 milliards de kilowattsheure, ne représente que $3,5 \%$ permettant d'économiser $50 \%$, alors que dans le projet de canal, la perte de cet ordre prévue est de $150 \%$. La mesure s'impose donc. L'étanchement de la superstructure de la digue sera assuré par un moyen connu cinq fois moins coûteux que l'emploi des palplanches métalliques.

En estimant la perte par infiltration trois fois plus faible dans le fleuve canalisé que dans le canal, par suite du retour aux biefs aval de l'eau pérdue par les biefs amont (chose défendue à la solution par canal), on considère en fait un état de porosité, sur les onze biefs prévus, correspondant à 55 , au lieu de 15 dans le canal : on ne pourra donc pas nous taxer d'exagération!

Etant donnés les avantages de tous ordres assurés par la canalisation du fleuve, on pourrait tout aussi bien admettre le colmatage superficiel de son lit, ainsi que la chose s'impose pour le canal.
Mais on devrait alors abandouner la solution si intéressante de la largeur du fleuve portée à 600 mètres.

En s'en tenant à la largeur actuelle de 200 mètres du lit approfondi, le bétomnage du fond n'aurait que 16 mètres de plus de largeur de revètement que celui prévu pour le canal, chose insignifiante. En temps d'eau ordinaire, on réaliserait le travail en deux fois : l'approfondissement exécuté, on diviserait le lil en deux parties égales par un masque longitudinal en palplanches métalliques amovibles, done ensuite réutilisables alleurs. Le travail effectué à sec, le lit aurait une régularité définitive, puisqu'on n'aurait plus à y redouter l'action ćrodante des principes lourds en mouvement, le fleuve n'en recevant pas de l'amont et n'en empruntant plus à son propre fond, outre que la vitesse de l'eau, mème en temps de crues, restera en dessous de celle acceptie dans le canal.

Mais toute filtration souterraine sera empêchée par le masque en palplanches métalliques que nous proposons d'établir sous les digues; d'où la possibilité de consacrer le principe de la laryenr maximum des biefs de 600 mètres. Si on peut se tenir à l'ćpaisseur que nous proposons pour ce masque, il résulte des renseignements que nous avons recueillis que le prix de ce masque sera sensiblement celui prévu. Dans la pratique, on a eu à enfoncer ces palplanches par 22 mètres de profondeur, limité à cet égard par la solution envisagée et non par des difficultés technicques. Nous avons eu à enfoncer des rails, en vue des sondages de Génissiat, par 31 mètres de fond. Mais il semble acquis que la masse étanche doit se rencontrer à 5 ou 6 mètres de profondeur. Pour ètre fixé à ce sujet, il en coûtera peu de procéder d'après la míthode que nous avons préconisée des puits instantanés : a l'aillo d'un pieu à vis creux et métalliçue, on observera le débit de l'eau aspirée à mesure de la descente du pieu; une fois les masses colmatantes atteintes, le débit sera nul et la longueur des palplanches définie.

A remarquer que la solution que nous proposons permet d'ancrer les bateaux en tous points, tandis que le bétonnage, dans les deux cas, exigerait l'application en surface d'une masse meuble importante, encombrante et difficile à maintenir régulière.

Afin de demander à ce voile d'empècher les sous-pressions si dangereuses pour les digues, on l'établira sous l'accotement formant la crête de la digue du côté de l'eau.

Comme il importe que les travaux soient effectués à l'aide des moyens les plus expéditifs, il faut que les dispositions prises s'y prètent. Or, ces ouvrages s'exécutent ordinairement en trois ou quatre campagnes au plus. Si on gagne un an, c'est 8 milliards de kilowatts-heure d'une valeur brute de près de 400 millions en or matériellement récupérés, sans compter les avantages relatifs à la navigation, aux irrigations et à la suppression des inondations.

Le fleuve aménagé par notre méthode présentera une ligne de défense plus copieuse qu'actuellement et à sa place légitime avec un fossé d'eau de 600 mètres, au lieu, pour le canal, d'un fossé, asséché le premier jour, de 120 mètres de largeur n'ofirant qu'une défense illusoire sur notre territoire.

L’éloignement presque général de tout lieu habité sur les surfaces à occuper par la largeur de 600 mètres des biefs que nous proposons, favorise cette heureuse disposition.

Le canal, en temps de crues, n'aurait aucune utilité locale au point de vue de la navigation, faisant alors double cmploi avec celui du Rhône au Rhin qui lui est proche et parallèle sur loute sa longueur.

\section{ORGANISATION DE L'ENTREPRISE GÉNÉRALE}

On aura à créer immédiatement un bureau central chargé de l'étude générale de l'aménagement. La documentation existante sera demandée aux services compétents cles pass riverains, dont les ingénieurs devront participer à l'étude faite ainsi en conmun, pour ne laisser aucun intérêt dans l'oubli. Toutes les difficultés d'ordre polilique ou technique seront arbitrées par la cour de justice du Tribunal international de La Haye. 
On ćtablira d'abord un projet d'ensemble pour la répartition judicieuse de l'emplacenent des ouvrages. Puis l'étude pour chaque bief fera l'objet, au besoin, d'une mise au concours, comme la chose a été laite sur le Rhin supérieur, afin d'assurer à cette étude une discussion approfondie et donner la plus grande somme de satisfaction aux intérêts en cause. Pour ne pas perdre de temps, ce travail de commission se fera concurremment avec celui des levers préalables, des études géologiques, hydrologiques et financières de chaque partic, des expropriations, etc. Puis on mettra en adjudication, séparément pour chaque bief :

$1^{\circ}$ Le dragage général et l'exécution des digues suivant la méthode choisie;

20 La réalisation sur bassin de radoub des éléments en ciment armé de toutes les parties des ouvrages;

$3^{\circ}$ La mise à flot dans la cale de radoub, le transport sur place et l'échouement à la place requise de ces éléments;

4. La réalisation en ciment armé de toute la superstructure des barrages, ponts, bâtiments d'usines, bureaux, ouvrages dans les digues, déversoirs et reversoirs, ponceaux, etc.

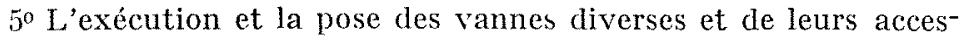
soires, appareils de levage, grilles avec dégrilleurs, etc.;

$6^{\circ}$ L'établissement des turbines et de leurs accessoires mécanicques ;

$7^{\circ}$ L'exécution des dynamos, transformateurs, etc. ;

$8^{\circ}$ Les lignes électriques raccordant toutes les usines des barrages, aux fins de suppléance, et celles servant aux distributions locales et régionales;

go Les lignes internationales de transport et de distribution de l'énergie disponible, lorsque les besoins locaux et régionaux seront assurée ;

$10^{\circ}$ La réalisation d'un moyen pratique, à poste fixe dans le fleuve, de halage rapide et économique des bateaux.

Gràce à une entente avec la Suisse en voie de réalisation, le remous du barrage à étudier vers Huningue va être porté au delà de Bàle, jusqu'au barrage futur de Birsfelden.

L'ensemble des onze barrages à prévoir serait à établir à l'aval de Bâle dans l'ordre et avec les caractéristiques ci-après, se partageant les 134 kilometres du parcours et les 114 mètres de pente brute dont 7 kilomètres et 7 mètres empruntés au remous suisse et au dragage à l'aval du dernier barrage.

\section{Tableau II}

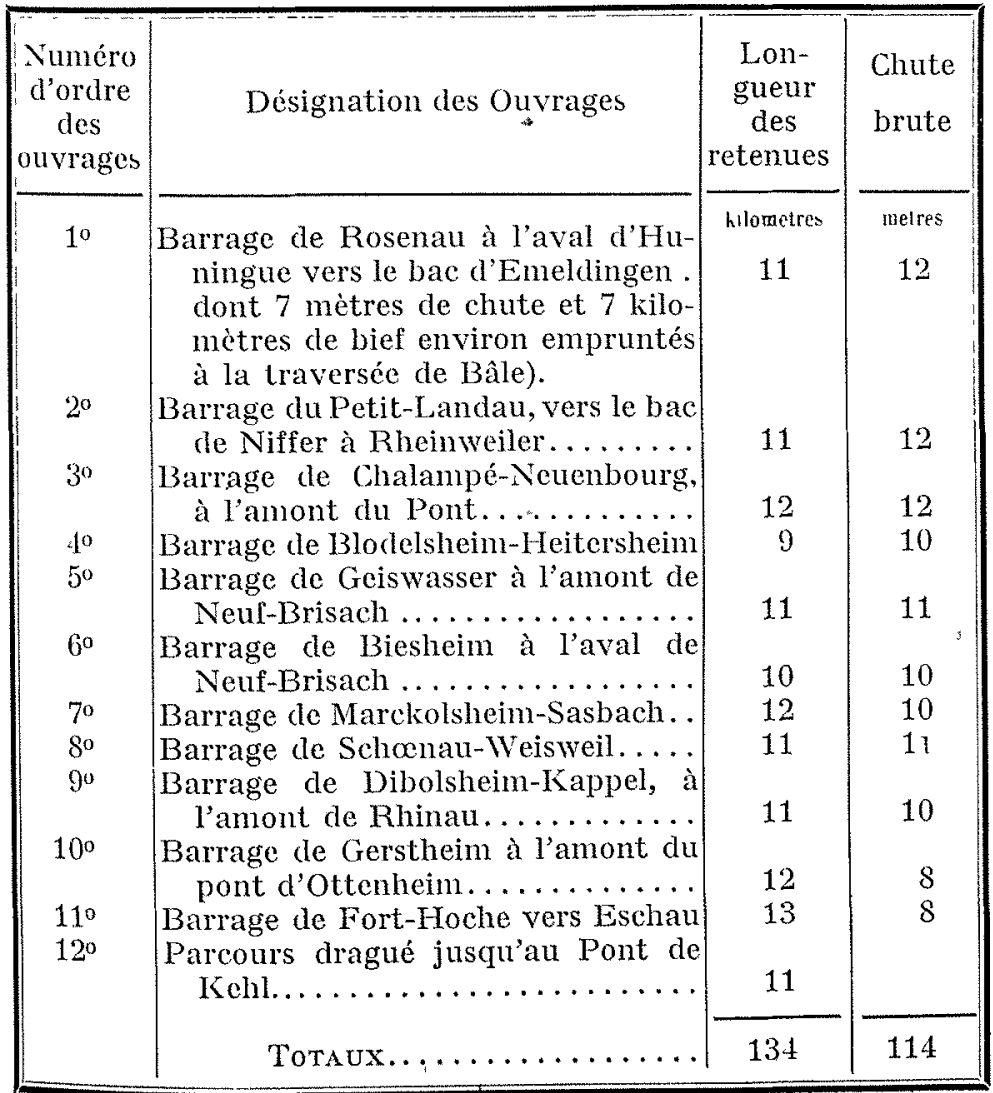

On voit que, pour des biefs peu nombreux, les hauteurs des ouvrages de retenue sont des plus favorables à la récupération économique de l'énergie de l'eau.

L'aménagement jusqu'à Lauterbourg, pour une pente de 0 mo 51 par kilomètre, comporterait les ouvrages ci-après :
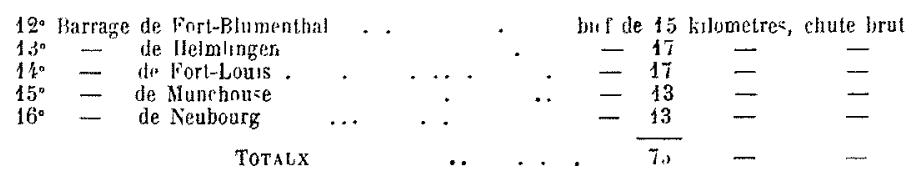

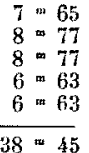

Le contingent fourni au Rhin par les eaux territoriales allemandes, de régime jurassique pour la plupart, est d'un quart pendant l'été et des trois cinquièmes durant l'hiver. Dans ces conditions, on voit tout l'intérêt à améliorer la régularisation à l'aide des lacs suisses dont les eaux sont de régime glaciaire, et à ne pas perdre le bénéfice de l'utilisation du débit des affluents de rive droite entre Bâle et Strasbourg, puis de I'utilisation de la pente jusqu'à Lauterbourg devant grandement améliorer le régime d'hiver de la force motrice.

\section{ÉTABLISSEMENT DES USINES HYDRO-ÉLECTRIQUES}

On aura intérêt à réduire le plus possible le nombre des unités hydro-électriques, en leur donnant une vitesse de rotation maximum.

Les turbines seront du type à axe vertical et libre déviation, qui se prête le mieux aux grands débits. Elles seront disposées pour rester norées bien qu'en aspiration en dessous, même quand les crues donneront le maximum de relèvement du plan d'eau; la hauteur de chute sera relativement maintenue par la majoration du niveau de la retenue, l'accroissement du débit compensant éventuellement la moindre pression.

On installera immédiatement le nombre d'unités voulu pour I'utilisation d'un débit de 1.000 mètres cubes d'eau en moxenne, avec faculté de porter ce débit au double aux heures de pointe. Si une entente est réalisée avec les producteurs d'énergie du Rhin supérieur, il suffira de demander au Boden-See l'appoint d'eau voulu pour que, de barrage à barrage, l'accroissement du débit s'effectue, les biefs ne pouvant voir leur plan d'eau sensiblement diminuer, puisque chacun d'eux sera, à mesure, suralimenté par le bief amont. Seule, la vitesse de l'eau sera accrue, mais d'une facon acceptable par la navigation.

Toutes les usines hydro-électriques seront raccordées, aux fins de rendre très élastique la production et l'utilisation de leur énergie ainsi groupée en vue de grands transports européens à très haute tension très économique,

\section{NAVIGATION GÉNERRALE ET HALAGE ÉLEGTRIQUE DES BATEAUX}

La grande navigation pouvant comprendre même des chalands de mer naviguant par leurs propres moyens, et ceux des compagnies de navigation employant des transporteurs automoteurs. Il va de soi que le halage électrique proposé n'offrira qu'un avantage facultatif, et que tous les modes de navigation seront admis dans les biefs, même celui à voile. Pour le halage électrique, des lignes doubles pourront être établies longeant le bord de la rive droite. Ces lignes seront constituées à l'aide de deux monorails superposés à crémaillère, le monorail inférieur disposé à 4 ou 5 mètres de hauteur au-dessus de la rive portant les tracteurs des bateaux montants longeant la rive, alors que le monorail supérieur, disposé à 1 mètre plus haut, servira de piste aux tracteurs menant les bateaux descendants et passant au large. L'ensemble de ces monorails et les lignes de trolleys de prise de courant seront supportés par un câble caténaire établi sur des pylònes espacés de 50 à 100 mètres, le tout convenablement contreventé pour réagir contre les efforts en plan.

La commande des tracteurs électriques se fera à distance et résultera au départ de la seule tension de la remorque à la main par le batelier; d'où une économie de personnel, le pilote suffisant où l'emploi d'un wattman à terre et au moins deux hommes sur les remorqueurs s'imposent avec les autres méthodes.

Si la largeur des biefs de 600 mètres est adoptée, rien ne s'opposera à ce que la ligne de halage soit implantée dans le lit, tout proche du lit mineur offrant le plus de mouillage aux bateaux. 
Les pylònes seront ainsi a l'abri du choc des lourds bateaux qui ne s'expliquera, du reste, plus sur un fleuve alors presque sans courant.

Ce sera une ressource pour réaliser un balisage copieux et l'éclairage pour la navigation nocturne, fluviale et aćriemne des hydravions suivant par sécurité le lit du Rhin.

A la faveur d'une grande rapidité offerte aux bateaux de touristes, il est possible d'entrevoir un certain intérèt à assurer un service spécial pour les voyageurs sur cet itinéraire si vanté. Afin de rendre indépendant ce service, il nous parait réalısable d'établir une ligne double, à son usage, et disposée sur les mèmes pylònes que ceux destinés aux lignes de halage des chalands. Ces bateaux à voyageurs, d'un mouillage réduit, se satisferont du tirant d'eau qui sera assuré en tout temps entre la digue de rive droite et la partie approfondie et où la vitesse du courant sera minime.

Ce mode de halage électrique a été jugé, par des constructeurs très experts, comme trois à six fois plus économique d'installation et de fonctionnement que tout autre présentant les mêmes avantages de commodité et de robustesse. Le trématage s'effectue sans rompre charge gràce à la superposition de la circulation des tracteurs offrant ainsi une voie double à peu de frais.

Le moyen Flettner, quinze fois plus avantageux à section égale exposée au vent que la voile, trouvera aussi son domaine d'emploi, grâce au faible courant, à la condition de pouvoir faire osciller les cylindres verticaux servant à la propulsion au passage sous les ponts.

Il paraît tout indiqué, sitôt le principe de la canalisation du fleuve admiss, qu'on aura un avantage exceptionnel à établir immédiatement le halage électrique proposé qui sera au besoin alimenté d'énergie naturelle par du courant venant de Bâle. Outre une navigation copieuse, assurée, on animera à l'aide de la même ressource tous les engins de réalisation des travaux.

\section{PISCICULTURE}

Le problème de la pisciculture apporte un gage également intéressant à l'élargissement de la retenue dans l'aménagement du fleuve. On a estimé à 1.500 francs le produit, à l'hectare, des étangs qui furent aménagés avant la guerre pour tirer un parti rationnel des eaux usées de la ville de Strasbourg dont le Gouvernement allemand avait prohibé le déversement dans l'Ill. Les 200 kilomètres de fleuve, dont nous proposons l'aménagement de Bâle à Lauterbourg, représenteront donc, avec 600 mètres de largeur, 12.000 hectares, soit une ressource matérielle de 18 millions en or à en tirer par les fermiers de la pêche, surtout en tirant parti de l'emploi des lônes. Chaque barrage sera muni d'une échelle à poissons, et un institut international de pisciculture sera établi à Strasbourg.

\section{IRRIGATIONS}

L'eau en potentiel sur chaque barrage, sans l'établissement de têtes mortes coûteuses, sera conduite latéralement par la voie la plus courte suivant la pente naturelle, partie à l'aide de canaux, le reste en canalisations forcées. Le volume utile sera donné à discrétion pour l'alimentation d'un nombre considérable de municipalités, et tenir en eau la multitude de ruisscaux et canaux d'arrosage dont on voit la vallée sillonnée d'une façon merveilleuse, mais tous à sec au moment où leur concours devient le plus précieux. Or ce volume d'eau ne coûtera rien à la production de la force motrice, puisqu'il est représenté par le diagramme (fig. 1) sous la forme résiduaire ou sans affectation. Avantage évident sur l'aménagement par canal. Or la surface ainsi intéressée à ces irrigations peut être estimée à plus de 2.000 kilomètres carrés sans compter celles à alimenter par des refoulements acceptables. Or 200.000 hectares en période de sécheresse demandent le même nombre de litres à la seconde pour le maintien des irrigation, soit 200 mètres cubes. Que sera-ce pour le débit du fleuve, supérieur à 1.500 mètres cubes de mai à août, et comment ne pas être impressionné par cette considération en faveur de notre mode d'aménagement!

Il sera entendu que les syndicats d'irrigation auront à payer un droit d'eau équivalent au moins à la valeur de l'énergie non récupérée sur les turbines.
Les déversoirs el reversoirs seront d'un fonctiomement automatique en temps de crues et de décrues.

\section{L'AMENAGEMIEN'T DES GRANDS FLIUUVES EST' UN PROBLEME INTERNATIONAL.}

L'entente économique entre les peuples, sûr garant de la paix universelle, justifiera la réalisation la plus favorable possible de la grande navigation internationale aussi bien en mer que sur les grands cours d'eau. Comment attirer les produits des importateurs mieux qu'en leur rendant accueillants d'une façon avantageuse tous les points du territoire! D'où tout pavillon doit avoir, sinon acquérir. le droit de remonter le Rhin, le Rhône, l'Elbe, comme la Tamise. Il n'y a que des économistes à courte vue qui peuvent nier cette vérité si évidente.

Le rôle de la diplomatie est d'apprécier en outre le fait qu'il existe des ressources en forces naturelles à mettre sur le marché européen dans les conditions les plus favorables pour le bien de tous.

Ce marché sera praticable, si des droits de circulation ne viennent le paralyser. Or il est facile de justifier qu'on peut faire fonds sur une puissance de production disponible de l'ordre de 30 à 40 milliards de kilowatts-heure par an autour d'un point qui serait sensiblement Strasbourg, et provenant des forces du Rhône, du Rhin, des Alpes et de la Suisse, sans compter les ressources qu'il convient d'ajouter très importantes d'énergie scandinave.

Nous avons pleinement justifié en son temps l'avantage économique d'un transport d'énergie des Alpes à Paris d'une longueur de 500 kilomètres sous une tension de 100.000 volts et une perte moyenne en ligne limitée à $10^{\circ} \%$ qui va ètre bientôt une réalisation (1). Aujourd'hui, la tension de 220000 volts est pratiquement admise et celle de 300.000 volts certainement à envisager.

Si le rayon d'action, prévu alors dé 500 kilomètres, est porté au double, soit 1.000 kilomètres, avec une tension triple, doù une perte tröis fois moindre en ligne, mais une dépense de ligne clouble, augmentant d'un tiers la dépense générale ; et si on décompose le prix maximum de vente en gros à Paris de $0 \mathrm{fr}$. 05 en une partie constante de $0 \mathrm{fr}$. 03 et ane variable effective aux frais de transport, cette variable sera doublée par la distance, doublée encore par la perte en ligne, mais réduite au tiers, par la majoration dn la temsion. D'où le prix de revient, alors supputé, du kilowatt-heure transporté, de $0 \mathrm{fr} .05$ en or, deviendra de

$$
0.03+\left(\frac{0,02 \times 4}{3}\right)=0 \mathrm{fr} .06
$$

en chiffre rond ; porté à 0 fr. 065 pour tenir compte du prix majoré des supports, ce prix serait celui maximum de la vente en gros sur les points les plus éloignés, dans un rayon supposé au maximum de 1.000 kilomètres. Il est facile d'admettre que, tout en étant alors grevée de frais de distribution assez élevés, cette force hydro-électrique pourra y concurrencer avec avantage l'énergie thermique, dans la plupart des cas, surtout dans les régions dépourvues de houille.

Or, la distance en ligne droite du centre d'action, Strasbourg, pour atteindre les principales villes de l'Europe, est, pour Barcelone, de 950 kiloniètres, pour Rome, 890 kilomètres, Londres, 690 kilomètres, Vienne, 680 kilomètres, Berlin, 650 kilomètres, Amsterdam, 190 kilomètres, Paris, 422 kilomètres et Bruxelles, 380 kilomètres.

Aujourd'hui, où le problème de la synthèse du pétrole semble résolue, on ne voit pas comment on pourra se passer à tout jamais d'une ressource concrète en carbone pour cles usages mobiles et indépendants de l'énergle comme la locomotion libre et la grande navigation. En conséquence, si naguère on estimait la durée des gisements houillers anglais à cent cinquante ans, il convient de réduire d'un tiers cette période d'épuisement, par suite des besoins nouveaux s'accusant chaque jour. D'où un immense intérêt à ménager les réserves en combustible de notre continenl et s'adresser dans toute la mesure possible à la ressource inépuisable, bien que limitée, de l'énergie naturelle. Ceux qui ne voient pas tout l'intérêt de ce qui précède ont un entendement déplorable de la pérennité de la vie des nations. Ce sont les mêmes qui ne veulent pas voir que la première condition de l'établisse-

(1) Aménagemenl du Rhône, projet Blondel, Harle et Mähl 
ment de la paix en Europe esl celle d'une entente économique plus on donnera de gages à cette dernière, plus étroite elle deviendra ; et quand l'intérêt commande, on est toujours prêt à s'entendre !

Il conviendra donc de former un syndicat international, en vue de tirer le meilleur parti possible de cette formidable ressource en Europe et d'en assurer la production la plus logique et avantageuse en tenant compte de tous les intérêts en cause, notamment en ce qui concerne la réserve des lacs pour l'assurer la plus souple et abondante possible. Il devra porter son intérêt sur la réalisation d'un grand nombre de retenues des eaux résiduaires dans des lacs artificiels dont on conçoit en maints endroits la création très économique.

\section{AVANTAGES DIVERS DE L'ÉLARGISSEMENT DU FLEUVE}

\section{PROPOSE}

La largeur de 600 metres proposée ne joue aucun rôle indispensable au point de vue de l'écoulement des crues les plus grandes comme les calculs le démontrent. Annuellement inondées, il n'y a aucun inconvénient à incorporer des surfaces dans la retenue à laquelle elles donneront une ampleur pleine de ressource, à des points de vue multiples, puisqu'on pourra même y pratiquer la navigation à la voile, constituer un port continu sur les deux rives, réaliser la pisciculture en grand, etc...

Certains redouteront l'épanchement d'un grand volume d'eau en cas de rupture d'une digue : mais le mode de réalisation que nous proposons retire toute crainte à ce propos. Au pis aller même, si une digue cédait, pour un des biefs d'une longueur moyenne de 11 kilomètres et un déversement supposé même de la demihauteur maximum de la retenue latérale, soit de $\downarrow$ mètres, par une brèche qui mettrait un certain temps à s'agrandir, la tranche d'eau, en écoulement relativement ralenti, viendrait franchir la cunette latérale où elle existera presque partout naturellement, puiscue la vallée est remplie de lònes profondes se succédant sans cesse, sur chaque rive; ces lônes emplies, l'eau s'exhaussera sur le sol avoisinant dans des conditions très limitées, car, sitôt l'accident signalé aux barrages d'amont, on fermera successivement toutes leurs vannes dans la mesure nécessaire et réalisable pour diminuer momentanément le flot passant par le bief accidenté ; chose possible alors, même en temps de crues, où l'effet de la retenue s'étendra juscqu'à Bodensee. D'où l'eau épandue trouvera immédiatement une surface au moins dix fois plus grande, et la ressource de n'y produire qu'une crue de $0^{\mathrm{m}} 50$ au lieu de plusieurs mètres actuellement en temps d'inondations annuelles.
Mieux encore, un avertisseur sonore ad hoc signalant une baisse d'eau anormale sur le bief accidenté vers son barrage, les vannes de ce dernier seront rapidement soulevées et l'effet de déversement immédiatement enrayé, quel que soit alors le débit du fleuve.

De plus, puisque la commande des vannes sera électrique, il sera loisible de leur assurer un fonctionnement automatique dans cette circonstance. D'où une sécurité qu'on ne saurait offrir aussi grande dans le voisinage de tout autre cours d'eau endigué, et pour la vallée du Rhin une quiétude qu'elle n'a jamais eue.

A cela, il convient d'ajouter que les localités exceptionnellement proches du Rhin sont sur des surfaces dominant le fleuve et rarement atteintes par les débordements.

II va de soi que ce concours de l'ensemble des barrages sera précieux en toutes circonstances telles que les crues exceptionnelles d'affluents.

L'aménagement par canal, au contraire, fera empirer la situation, comme nous l'avons dit.

Le mode d'exécution des digues que nous proposons les mettra à l'abri de toute sous-pression. Il n'est guère d'exemples de digues à charge permanente partant instantanément sans que leur solidité ait été au préalable altérée, chose révélée par des fuites. Comme le grand mouillage offert aux bateaux ne sera pas utilisé de longtemps, celui assuré à l'aval de Lauterbourg ou de Strasbourg devant rester assez limité puisque aucun approfondissement n'y est prévu, il sera loisible, sans grand inconvénient, de donner aux digues une charge graduelle qui en démontrera la bonne exécution à la faveur d'une longue expérience. On prendra souci également de la modification aussi réduite que possible de l'état hydrologique dans les vals du Rhin, chose dont on sera maître par la profondeur des cunettes prévues longeant le pied du talus extérieur des digues; ou en observant les lônes dont le niveau et le débit vers I'aval seront fonction d'assourcements venant du fleuve les transformer à la longue en abîmes, quand ces lônes ne seront pas alimentées par des affluents sous forme directe ou de résurgences.

Le tableau ci-dessous donne les caractéristiques concernant les installations actuelles et futures par barrages successifs du Rhin supérieur.

La puissance maximum étant de $8+6.550$ chevaux et celle installée de 777.000 chevaux, cette dernière sera donc en surcharge de $9 \%$ en temps de crues durant lequel l'énergie maximum sera produite sur l'ensemble des aménagements. Nous comptons donner plus d'élasticité à l'utilisation du débit par les groupes électrogènes et accuser davantage l'utilisation de l'énergie résiduaire dans nos installations.

Tableau III

\begin{tabular}{|c|c|c|c|c|c|c|c|c|c|}
\hline \multirow[b]{2}{*}{ Situation } & \multirow{2}{*}{$\begin{array}{l}\text { Longueur } \\
\text { des } \\
\text { retenues } \\
\text { en } \\
\text { kilomètres }\end{array}$} & \multicolumn{2}{|c|}{ Hauteur de Chute } & \multirow{2}{*}{$\begin{array}{l}\text { Débit } \\
\text { maximum } \\
\text { utilisé } \\
\text { prévu en } \\
\text { mètres } \\
\text { cubes d'eau }\end{array}$} & \multicolumn{3}{|c|}{ Puissance en Chevaux } & \multirow{2}{*}{$\begin{array}{c}\text { Puissances } \\
\text { en } \\
\text { unités et } \\
\text { force des } \\
\text { turbines } \\
\text { en } \\
\text { chevaux }\end{array}$} & \multirow{2}{*}{$\begin{array}{l}\text { Puissance } \\
\text { totale } \\
\text { en } \\
\text { chevaux }\end{array}$} \\
\hline & & $\begin{array}{c}\text { en } \\
\text { basses } \\
\text { eaux }\end{array}$ & $\begin{array}{c}\text { eaux, } \\
\text { crues, } \\
1 \text { mètre à } \\
\text { Bàle }\end{array}$ & & Maximum & Minimum & $\begin{array}{c}\text { assurée } \\
\text { pendant } \\
200 \\
\text { jours }\end{array}$ & & \\
\hline Birsfelden... . & 8,7 & 7,72 & 6,24 & 1.000 & 67.000 & 19.900 & 50.700 & $12 \times 5.000$ & 60.000 \\
\hline Augst-Wyhlen .. & 7,0 & 7,46 & 6,81 & 700 & 56.100 & 18.000 & 45.000 & $20 \times 2.800$ & 56.000 \\
\hline Rlieinfelden..... & 5,4 & 8,48 & 8,20 & 1.000 & 89.500 & 20.000 & 69.200 & $11 \times 6.000$ & 66.000 \\
\hline Schworstadt.... & 12,8 & 11,85 & 11,52 & 1.000 & 131.400 & 27.900 & 102.600 & $10 \times 12.000$ & 120.000 \\
\hline Sackingen... & 8,7 & 6,40 & 5,57 & 1.000 & 62.500 & 15.100 & 47.100 & $12 \times 5.000$ & 60.000 \\
\hline Laufenbourg ... & 8,8 & 10,94 & 9,91 & 720 & 81.000 & 25.200 & 69.500 & 5.500 & 55.000 \\
\hline Dogern ........ & 12,0 & 11,15 & 10,62 & 810 & 91.000 & 27.300 & 77.200 & 9.500 & 85.500 \\
\hline Koblenz. Kad... & 10,8 & 11,30 & 10,76 & 420 & 47.600 & 13.500 & 37.200 & 8.000 & 40.000 \\
\hline Rekingen...... & 11,5 & 9,43 & 8,81 & 425 & 41.500 & 11.400 & 31.300 & 8.000 & 40.000 \\
\hline Eglisau.... & 19,7 & 15,77 & 10,75 & 330 & 40.000 & 12.900 & 34.200 & 6.500 & 45.500 \\
\hline Rheinau ... & 10,4 & 11,20 & 10,85 & 317 & 41.700 & 11.700 & 30.000 & $5 \times 8.000$ & 40.000 \\
\hline Rheinfall... . & 3,4 & 26,70 & 24,99 & 245 & 65.300 & 22.900 & 64.600 & $5 \times 15.000$ & 75.000 \\
\hline Schaffhausen.... & 16,0 & 8,23 & 5,22 & 360 & 23.000 & 5.900 & 16.000 & $6 \times 4.000$ & 24.000 \\
\hline \multirow[t]{2}{*}{ Rheinklingen ... } & 30,8 & 3,77 & 2,71 & 340 & 8.950 & 3.400 & 6.000 & $5 \times 2.000$ & 10.000 \\
\hline & & & & & 846.550 & 235.300 & 680.600 & & 777.000 \\
\hline
\end{tabular}


Tableau IV

Usines entre Bâle et Strasbourg sur biefs dragués et digues sur ilevées

\begin{tabular}{|c|c|c|c|c|c|c|c|c|}
\hline \multirow{2}{*}{ Barrages Usines } & \multirow{2}{*}{$\begin{array}{l}\text { Chute } \\
\text { moyenne } \\
\text { constante }\end{array}$} & \multicolumn{3}{|c|}{$\begin{array}{l}\text { Débit moyen utilisé } \\
\text { en mètres cubes }\end{array}$} & \multicolumn{3}{|c|}{$\begin{array}{c}\text { Puissance brute des usines } \\
\text { en chevaux }\end{array}$} & \multirow{2}{*}{$\begin{array}{l}\text { Production } \\
\text { moyenue } \\
\text { annuelle } \\
\text { en } \\
\text { kilowatts-heure }\end{array}$} \\
\hline & & $\begin{array}{l}\text { Quatre } \\
\text { mois }\end{array}$ & $\begin{array}{l}\text { Quatre } \\
\text { mois }\end{array}$ & $\begin{array}{l}\text { Quatre } \\
\text { mois }\end{array}$ & mininum & mojenne & maximum & \\
\hline 1. Rosenau.......... & 11.75 & 650 & 1.050 & 1.530 & 102.000 & 165.000 & 240.000 & $875,000,000$ \\
\hline 2. Petit-Landatu... . . . . . . & 11,75 & 660 & 1.050 & 1.530 & 101.000 & 165.000 & 210.000 & 878.000 .000 \\
\hline 3. Chalampé........... & 11,75 & 665 & 1.050 & 1.530 & 104.500 & 165.000 & $2 \cdot 10.000$ & 878.000 .000 \\
\hline 4. Blodelsheim........... & 9,75 & 670 & 1.060 & 1.530 & 87.000 & 138.000 & 199.000 & 719.000 .000 \\
\hline 5. Geiswasser . . . . . . . . & 10,75 & 675 & 1.060 & 1.530 & 96.500 & 152.000 & 220.000 & 813.000 .000 \\
\hline 6. Biesheim............ & 9,75 & 680 & 1.070 & 1.580 & 88.500 & 139.000 & 199.000 & 712.000 .000 \\
\hline 7. Marckolsheim.......... & 9,75 & 685 & 1.070 & 1.530 & $\$ 9.000$ & 139.000 & 199.000 & 7.12 .000 .000 \\
\hline 8. Schoenau ............ & 10,75 & 685 & 1.075 & 1.530 & 98.000 & 154.000 & 220.000 & 819.000 .000 \\
\hline 9. Diebolsheim........... & 9,75 & 690 & 1.075 & 1.530 & 90.000 & 134.000 & 199.000 & 731.000 .000 \\
\hline 10. Gerstheim ......... & 7.80 & 695 & 1.080 & 1.530 & 72.000 & 112.000 & 158.000 & 593.000 .000 \\
\hline 11. Fort-Hoche ........ & 7,80 & 700 & 1.080 & 1.530 & 73000 & 102.000 & 158.000 & $594.000 \quad 000$ \\
\hline Totaux & 111,35 & & - & & $1.00+.500$ & 1.575 .000 & 2.272 .000 & 8.390 .000 .000 \\
\hline \multicolumn{9}{|c|}{ De Strasbourg à Laulerbourg. } \\
\hline 12. Fort-Blumenthal........ & 7,15 & 705 & 1.085 & 1.530 & 70.000 & 108.000 & 152.000 & 573.000 .000 \\
\hline 13. Helmlingen........... & 8,50 & 715 & 1.095 & 1.535 & 81.000 & 124.000 & 174.000 & 066.000 .000 \\
\hline 14. Fort-Louis .......... & 8,50 & 725 & 1.105 & 1.540 & 82.500 & 126.000 & 175.000 & 682.000 .000 \\
\hline 15. Munchouse............. & 6,50 & 730 & 1.110 & 1.550 & 63.500 & 96.000 & 117.000 & 179.000 .000 \\
\hline \multirow[t]{2}{*}{ 16. Neubourg............ } & 6,50 & 740 & 1.120 & 1.550 & 64.000 & 97.000 & 134.000 & 512.000 .000 \\
\hline & $37,+15$ & & & & 361.000 & 551.000 & 752.000 & 2.912 .000 .000 \\
\hline Totaux généraux..... & 148,80 & & & & 1.365 .500 & 2.126 .000 & 3.024 .000 & 11.302 .000 .000 \\
\hline
\end{tabular}

Delxit̀ue Partie

Contribution à la discussion des projets d'aménagement du Rhin au moyen des deux méthodes proposées.

Les principales objections formulées par les rapports administratifs contre la canalisation directe du fleuve sont exposées ci-après avec les réponses qu'elles motivent. Les objections sont imprimées en italique.

$1^{\circ}$ On ne peut pas établir des barrages élevés dans la plane d' Alsace, le lut n'étant pas encaissé comme à l'amont de Bâle.

Avec notre méthode, cette critique ne se justifie plus gràce à l'approfondissement proposé. Disons toutefois, en ce qui concerne les digues nécessaires: qu'elles aient, pour une hauteur déterminée, à supporter la pression de l'eau du fleuve ou de l'eau du canal, dans les deux cas ces digues s'imposent. Mais dans notre projet, les digues, même avec une revanche d'eau facultative de 4 mètres que ne prévoit pas le canal, auront un relief sur la vallée de 4 mètres moins élevé que celui prévu pour les digues de canal (fig. 7). D'où un cube presque quatre fois moindre. On conçoit sans peine que le colmatage du fond qui s'impose pour le canal à une cote généralement plus élevée que celle du fleuve, ne se justifiera pas pour le lit mineur du fleuve approfondi, dont l'eau ne sera en potentiel qu'au voisinage des barrages sur des digues copieusement étanchées par les palplanches. Or les précédents de barrages et digues offrant une difficulté analogue, sans peme vaincue, sont nombreux ! (Les barrages du Drac à Grenoble, de la basse Isère, vers Valence, de Pougny-Chancy sur le Rhône, etc...).

Notre méthode a raison amplement de la critique médiocre formulée contre la hauteur des barrages possibles dans le lit; et il est de toute évidence qu'on pourra tout aussi bien établir dix autres barrages sur le fleuve, de plus de 10 mètres de hauteur de retenue, à la suite de celui prévu à Huningue par les canalistes qui, ne pouvant en éviter la construction, la trouvent facilement réalisable avec 8 mètres de retenue. Mais grâce à l'approfondissement, nous pourrons, si nous le voulons, ne pas excéder la pression exercée sur les rives à Huningue daas le voisinage de tous nos barrages ! $2^{\circ}$ Les barrages seront difficiles à construire et à entretenir et seront exposés aux crues du lleuve.

Mais on a l'expérience de leur construction et de leur entretien: peut-on en dire autant d'un canal mixte dont le rôle est universellement condamné, parce que : $1^{\circ}$.peu praticable à la navigation à cause de la trop grande vitesse de l'eau, en écoulement : $2^{\circ}$ exigeant des dessablages annuels coûteux; $3^{\circ}$ des frais d'établissement et d'entretien des berges qui augmentent géométriquement avec la vitesse de l'eau ; to ne se prêtant pas à une majoration appréciable du volume d'eau à utiliser; $5^{\circ}$ ne pcrmettant pas des embâcles, plus accessible au gel ; $6^{\circ}$ enfin la comparaison que nous avons faite du capital à investir pour l'aménagement d'un kilowatt-heure suivant l'une ou l'autre méthode devant convaincre les plus prévenus contre la canalisation du fleuve.

La conséquence, pour une crue même de 5.000 mètres cubes, fort rare sur le Rhin, sera, pour des vammes l'une largeur totale supposée de 200 mètres au plus, pour chaque barrage, d'être soulevées de moins de la moitié de leur hauteur, soit de 5 mètres (1); ce qui, avec les équipements électriques de manœuvre des vannes Stonay, demandera quelques minutes. Comme on disposera alors de plus de la moitié de la hauteur de chute et cue cette hauteur sera compensée par une majoration de débit, on ne s'apercevra de la situation critique créée par la crue extraordinaire qui, même avec le débit de 5.000 mètres cubes, pourra être entièrement écoulée par la section endiguée comme le calcul le justifie, même si on ne réalise pas l'élargissement proposé.

Mais jusqu'à la mise en charge de chaque barrage, l'ouvrage en son entier, les pertuis des écluses en particulier, n'offriront pas plus d'obstacle à la circulation que la création d'un pont, sauf pour l'exécution successive des radiers des vannes offrant un encombrement tout au plus à la fois de un huitième de la largeur du lit du fleuve.

\section{$3^{\circ}$ Un grand nombre d'écluses à traverser, oulre le passage des sept ponts de bateaux.}

Les onze grands barrages prévus, offrant la ressource d'autant de grands ponts carrossables, feront disparaitre l'intérèt des sept

(1) Pour 200 metres de larpeur lolale des vames, on aum un débl do $200 \times 5$ $\times 0,62 \times \sqrt{29 \times 7,5}=7.500$ mòtres cubes a la seconde, solt moilie plus que le nceessane. 
ponts de bateaux, et la manouvre des écluses moins longue par notre moyen que celle nécessaire aux ponts de bateaux.

\section{$4^{\circ}$ La vitesse de l'eau sera, à l'amont des biefs, sensiblement la même qu'actuellement el gênante pour la navigation.}

Nous avons montré cette vitesse réduite, en temps de débit moyen de 1.150 mètres cubes, à $0 \mathrm{~m} 63$ à la seconde, dans la partic la plus réduite de notre canalisation à l'aval des barrages, alors que le dragage général aura èté effectué; la vitesse sera réduite à $0^{\mathrm{m}} 20$ environ à leur amont; mieux encore : ces chiffres seront réduits de moitié, si on endigue un lit majeur de 600 mètres de largeur. Dans le canal, la vitesse constante sur sa longueur serait donc le double ou le quadruple de celle à prévoir sur les hiefs du fleuve canalisé.

\section{$5^{\circ}$ En temps de crues, la navigation sera rendue difficile.}

Cettc critique ne s'applique pas à notre méthode : dans les grandes crues annuelles de 3.000 mètres cubes, on n'aura que $1^{\mathrm{m}} 40$ de vitesse de l'eau à l'amont des biefs, et moitié moins si on donne à ces bief's la largeur proposée de 600 mètres, vitesse deux fois moindre encore vers les barrages; alors que dans un canal, on envisage une vitesse sur toute sa longueur, de $1 \mathrm{~m} 20$. D'où, avec presque deux fois moins d'eau employée, une vitesse moyenne de l'eau double ou quadruple dans le canal. Et même si, dans des cas exceptionnels, qu'on ne saurait prévoir même en se montrant aussi pessimiste qu'on voudra pour les calculs, on doit permettre à une partie de l'eau de circuler en dehors des digues sur son domaine actuel, on ne troublera pas, par ce fait, la navigation et la production de la force motrice sur chaque bief endigué, alors que la navigation sur le canal sera paralýée par son isolement aux issues vers un fleuve en crue.

$6^{\circ}$ Pourquoi parler de dragages dans les biefs du fleuve aménagé consécutifs à chaque crue?

Le fleuve ne charrie presque actuellement que son propre fond, rien à peu près ne lui venant de l'amont, les affluents étant généralement des émissaires de lacs suisses. Qu'aura-t-il à déplacer lorsque, par le grand dragage que nous proposons, on aura établi, si l'on veut, la courbe d'équilibre justifiće dans chacun des biefs? On n'est nullement gêné par les apports aux barrages alpms, en particulier à Chèvres, sur le Rhône, où passent cependant, sans nécessité de dragages, les 600.000 mètres cubes d'alluvions amenées annuellement par l'Arve.

Comme à Chèvres, on pratiquera la propulsion, à l'aval de chaque bief, des alluvions à la faveur de chasses d'eau effectuées une ou deux fois par an, le bief à dessabler préalablement affamé; si toutefois le courant pouvant atteindre plus de 2 mètres au fond, durant les très grandes crues, ne déplace pas ces alluvions en entier alors qu'une vitesse de $0 \mathrm{~m} 60$ généralement doit suffire à les faire circuler. Le curage, dès que les atterrissements se manifesteront, sera remplacé par les hersages, provoquant la mobilisation des alluvions dans le courant.

\section{$7^{\circ}$ On disposerait sur le fleuve de petules chules annihilées au temps de crues et donnant au total moins de force que le canal latéral.}

Notre méthode change tout cela.

On sait que les aménagèments par canalisation des fleuves sont généralement payants, surtout dès qu'on arrive à pouvoir utiliser la pente sur des barrages de 6 mètres de hauteur, plus que sur des clérivations mème relativement courtes; or nous proposons de donner de 8 à 13 mètres à la hauteur des retenues, chiffres que l'approfondissement général permettra peut-être de dépasser en vue de la réduction du nombre des biefs qu'on pourra peut-être envisager à la faveur d'une étude plus complète. Nous ne voyons pas pourquoi on sera plus audacieux dans l'adoption de plus grandes hauteurs de chute sur le canal où on rencontrera une difficulté beaucoup plus coûteuse d'empêcher les filtrations souterraines.

Au contraire, pour les digues du fleuve, celle du côté de la rive badoisc sera établie sur un sol en pente assez élevée et rempli de lônes pour lequel Ies filtrations moins chanceuses n'auront aucune importance. Du reste, le voile en palplanches métalliques étanchera copieusement la section jusqu'aux masses compactes argileuses reconnues dans le substratum. Tandis que le canal, forcément à une cote générale plus élevée que lo lit du fleuve ap- profondi, aura ses deux digues en perpétuel état préoccupant. Puis, la perte d'eau, définitive pour le canal, tandis que les filtrations reviendront toujours rendre l'eau dans les biefs inférieurs du fleuve aménagé.

Lorsque se produiront des étiages d'été, en perspective de plus en plus avec la régression bien constatée de la surface des glaciers, comment acceptera-t-on le prélèvement sur le minimum de $300 \mathrm{mè-}$ tres cubes des 200 mètres cubes d'eau à la seconde réclamés impérieusement par les irrigations, les besoins d'édilité, etc. ?

Quant à la question de la ressource en force motrice, la justification que nous avons faite de produire pratiquement plus de 11 milliards de kilowatt-heure, l'aménagement poussé jusqu'à Lauterbourg, au lieu de 5 milliards par le canal qu'on ne peut pratiquement prolonger au delà de Strasbourg (ou seulement 4 milliards 400 millions, maximum suivant nos estimations), montre que l'objection faite est sans fondement.

\section{So Le projet mixte de barrages et dérivations successives n'est pas} à retenir.

C'est notre avis, car on accroitrait la dépense dans des conditions injustifiées par les résultats à en attendre.

\section{$9^{\circ} L^{\prime}$ administralion s'estime liée par le Traité de Versailles en ce qui concerne la dérivation du fleuve.}

En vertu de cette théorie, on devra donc consacrer le principe d'une cuvre antiéconomique et antisociale! Mais l'article 358 du traité de paix spécifie que la France n'est pas affranchie de s'assurer que l'cuvre est rationnelle et susceptible de ne causer aucun préjudice aux autres Etats.

\section{$10^{\circ}$ On ferall acte antupatriotique et le jeu des ennemis si on rendat irréalisable le projet de canal latéral.}

Le soussigné s'honore de faire une cuvre patriotique de la plus haute importance en s'efforçant de réagir contre l'aveuglement de ceux qui, sciemment ou inconsciemment, s'évertuent à faire adopter une solution antiéconomique, déjà, par ce fait, en contradiction avec l'esprit du Traité de Versailles, et pouvant avoir les conséquences les plus épouvantables pour la tranquillité universelle dans l'avenir.

$11^{\circ}$ Pour la voie navigable, on n'a aucun intérèt à la voir réalisée entre Strasbourg et Lyon par la Suisse. Il convient, au contratre, de faire tous les efforts nécessaires pour améliorer la voie françalse.

Nous domnons d'autre part la justification de notre manière de voir à ce propos qui repose sur des considérations écononiques indiscutables pour la France et tous les Etats intéressés.

$12^{\circ}$ On ne peut dire que la France doil se désmtéresser de ce qui devra être fait à l'amont de Bâle : un problème d'une très grande importance y est attaché, celui de la régularisation du débit des lacs devant permettre une majoration sensible de la puissance d'étiage et, surtout, un soutien de poinle exceptionnel dans la variation du débit à l'aval du Boden-See dont toutes les usines du Rhin doivent bénéficier; comme il en sera sur le Rhône, si on domne à la grande usine à établir vers Génissiat le caractère que nous proposons de Iui octroyer, d'assurer non seulement la pointe saisonnière mais quotidienne pour l'ensemble des distributions françaises rattachées à la production sur le Rhône. Il y a tout à réaliser sur le haut Rhin comme sur le bas Rhin an mieux de l'intérèt général, et la France ne doit pas, au nom d'un sentiment étroit de ses droits, sacrifier cet intérèt économique supérieur qui est le sien en particulier tont en étant international.

Si les Allemands ont pu, à l'occasion, parler dẻ quatorze barrages à établir entre Bâle et Strasbourg, il n'est pas prouvé qu'ils repousseraient une solution ne comportant qu'une dizaine de retenues et même peut-être moins, en faisant état d'un grand approfondissement du fleuve à l'amont cle chaque bief comme nous le proposons; ce qui permettra de réduire en conséquence davantage le relief des retenues dont on redoute la pression de l'eau exercée favorisant les infiltrations.

(A suivre.) 